\title{
Un gigante reaparece: Danta Colombiana Tapirus terrestris colombianus (Perissodactyla: Tapiridae) en el Magdalena Medio antioqueño, Colombia
}

\author{
Andrés Arias-Alzate ${ }^{1,2 *(i)}$, Daniel Restrepo-Marín ${ }^{3}$ \\ 1 Programa de Ecología, Facultad de Ciencias y Biotecnología, Universidad CES, Calle 10A \# 22-04, Medellín, Colombia. \\ 2 IUCN/SSC Tapir Specialist Group (TSG) \\ 3 Corocora Wildlife Camp, Los Llanos, San Luís de Palenque, Colombia \\ *Correspondencia: aariasa@ces.edu.co
}

\section{Resumen}

El tapir de Tierras Bajas, Tapirus terrestris, es la especie de mayor distribución en Sur América. En Colombia presenta una distribución fragmentada, con tres subespecies: Tapirus terrestris terrestris, Tapirus terrestris aenigmaticus y Tapirus terrestris colombianus (la Danta Colombiana). Esta última subespecie endémica para el país. Gran parte de la distribución original para la Danta Colombiana se encuentra amenazada por la expansión de la frontera agrícola-ganadera y la cacería, por lo que se considera extinta en muchas localidades. Luego de 19 años sin reportes en el Magdalena Medio antioqueño, Colombia, documentamos los primeros registros que confirma su reaparición en la zona. Aunque estos registros parecen señalar una recolonización reciente del área, las bajas densidades poblacionales, producto de la presión antrópica, posiblemente hicieron que pasara desapercibida durante años. Las altas presiones antrópicas sobre los ecosistemas naturales, señalan la problemática que afronta la especie en términos de conservación en el futuro cercano en la región.

Palabras clave: Cámaras trampa, Distribución, Tapir, Tapirus terrestris colombianus, Valle del Magdalena Medio.

\begin{abstract}
The Lowland tapir, Tapirus terrestris, is the most widely distributed species in South America. In Colombia it presents a fragmented distribution, with three subspecies: Tapirus terrestris terrestris, Tapirus terrestris aenigmaticus and Tapirus terrestris colombianus (the Colombian Tapir). This last subspecies is endemic to the country. Much of the original distribution for the Colombian Tapir is threatened by the expansion of the agricultural-livestock border and hunting, which is why it is considered extinct in many localities. After 19 years without reports in Magdalena Medio, Antioquia, Colombia, we documented the first records that confirm its reappearance in the area. Although these records seem to point out a recent recolonization of the area, the low population densities, due to anthropogenic pressure, possibly made it go unnoticed for years. The high anthropic pressures on natural ecosystems, indicate the problems that the species faces in terms of conservation in the near future in the region.
\end{abstract}

Key words: Camera traps, Distribution, Middle Magdalena Valley, Tapir, Tapirus terrestris colombianus 
Tapirus terrestris es la especie más ampliamente distribuida del género en Sur América. Su rango de distribución abarca principalmente los ecosistemas de tierras bajas, desde los 0 hasta los 2438 msnm, desde el Norte de Colombia, Venezuela, Ecuador, Perú, Bolivia, Brasil, hasta el norte de Argentina y Paraguay (Hershkovitz 1954; Padilla \& Dowler 1994; Varela et al. 2019). En Colombia actualmente la especie presenta una distribución disjunta, con tres de las cuatro subespecies descritas: Tapirus terrestris terrestris hacia el oriente del país en la Orinoquia y Amazonía, Tapirus terrestris aenigmaticus hacia el suroriente del país y Tapirus terrestris colombianus en las tierras bajas del norte de Colombia con una distribución fragmentada (Hershkovitz 1954; Constantino et al. 2006; Arias-Alzate et al. 2009; Racero-Casarrubia \& Arias-Alzate 2015).

Actualmente la Danta Colombiana (Tapirus terrestris colombianus) se considera extinta localmente en Colombia, en muchas de las áreas de su distribución original (ver Hershkovitz 1954), entre ellas el Magdalena Medio antioqueño. Esto principalmente por la fragmentación y destrucción de los hábitats naturales debido a la expansión de la frontera agrícola-ganadera y su cacería indiscriminada (Arias-Alzate et al. 2009). Por ello, la especie se encuentra catalogada como Vulnerable (VU) según la UICN y específicamente Tapirus terrestris colombianus se encuentra catalogada en Peligro Crítico (CR) a nivel nacional (Constantino et al. 2006; Resolución 1912-2017 Minambiente; Varela et al. 2019).

Aquí documentamos los primeros registros que confirman la reaparición de la Danta Colombiana en las tierras bajas del Magdalena Medio antioqueño, Colombia. Estos representan un registro notable en un área, que en las últimas décadas ha sufrido grandes presiones antrópicas y una fuerte pérdida de los ecosistemas naturales (Arias-Alzate et al. 2013).

La localidad está ubicada a 112 m en el flanco occidental del valle del Río Magdalena, en la vereda San Bartolo, del municipio de Yondó, departamento de Antioquia. El paisaje está conformado por fragmentos de bosque primario y secundario, en una matriz de pastos para la ganadería extensiva (Figura 1A). De acuerdo con el sistema de clasificación de Holdridge (1947), esta área corresponde al bosque muy húmedo tropical (bmh-T). Los registros fueron obtenidos a partir de las actividades de seguimiento y control del territorio y monitoreo de la biodiversidad, específicamente durante las actividades de seguimiento al conflicto humanos-grandes felinos, desarrollado por la Corporación Autónoma Regional del Centro de Antioquia-CORANTIOQUIA.

Los registros se obtuvieron por medio del uso de cámaras de rastreo (Bushnell Trophy Cam Essential HD) dispuestas en modo video. En total se instalaron 8 cámaras de manera aleatoria a una distancia promedio de $700 \mathrm{~m}$, al interior de los fragmentos de bosque y a orillas de quebradas y potreros de la zona. Las cámaras permanecieron activas del 14 de junio al 24 de julio de 2016. Se ubicaron en árboles a aproximadamente $50 \mathrm{~cm}$ desde el suelo con la siguiente configuración: videos con alta resolución HD, sensor infrarrojo para día y noche, un video de 15 segundos por evento e intervalo de 10 segundos entre videos.

En total se obtuvo un esfuerzo de muestreo de 320 cámaras/noche, durante el cual se recopilaron 8 videos de la especie. Estos videos fueron registrados en 4 de las 8 estaciones de muestreo (Figura 1A, Tabla 1). Es importante mencionar la presencia en la zona, de al menos un macho y una hembra (Figura 1B). Esta identificación se realizó a partir del reconocimiento de los órganos reproductivos. Para el caso de la hembra es posible visualizar la presencia de mamas en su zona inguinal y en el macho es posible observar sus testículos desarrollados en su parte posterior (Figura 1B). Aunque cada video fue registrado con 
una separación e independencia de más de un día (Tabla 1), no es posible afirmar que los machos y las hembras sean los mismos individuos.
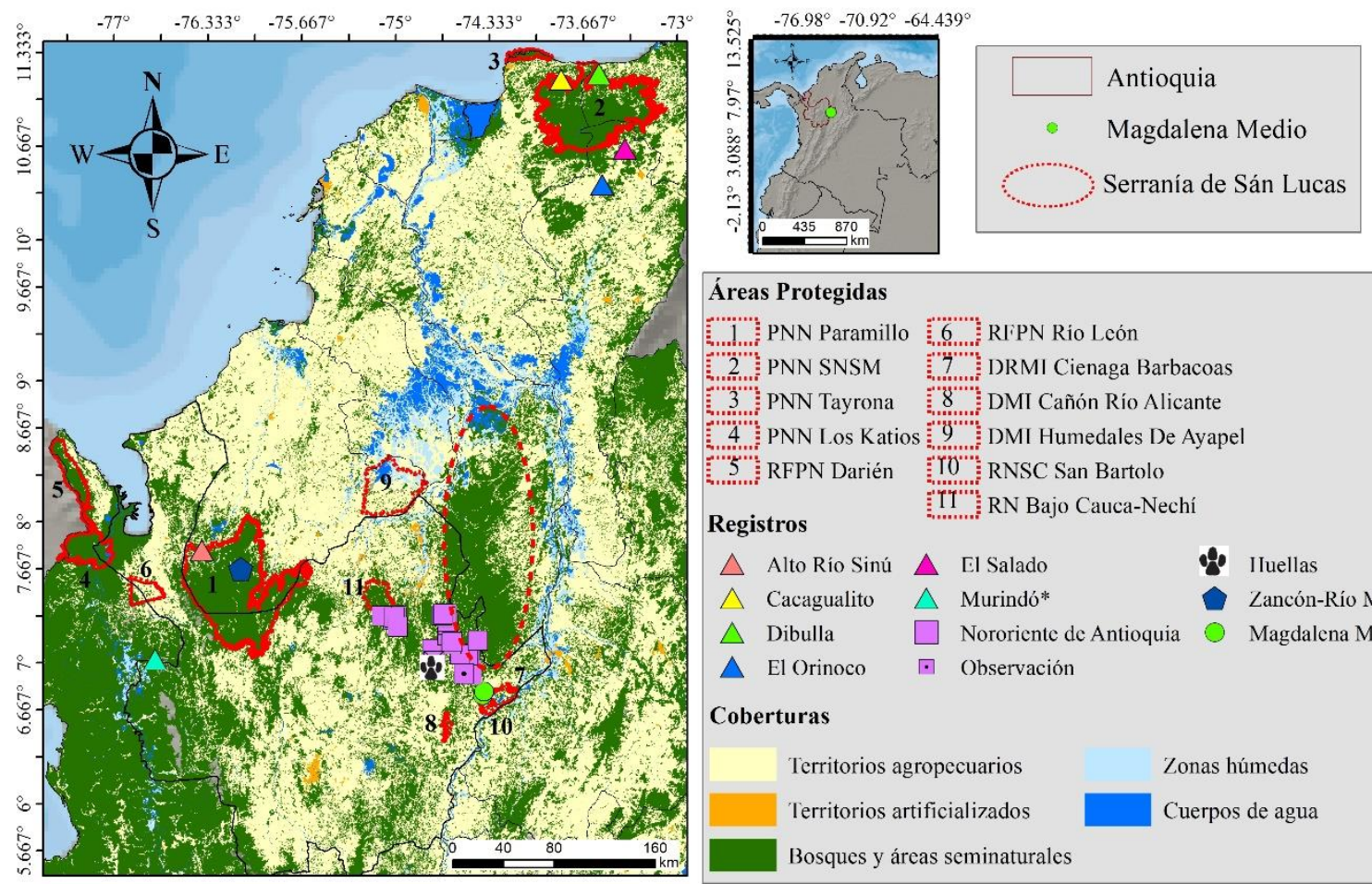

A

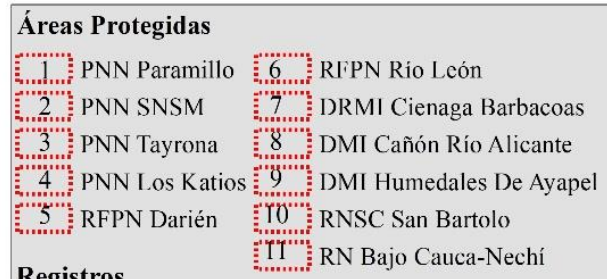

Registros

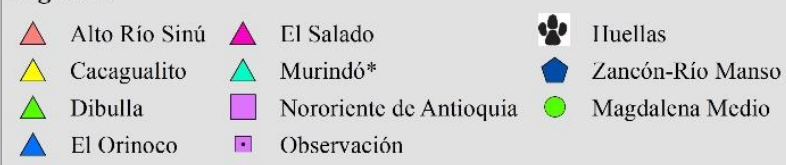

Coberturas
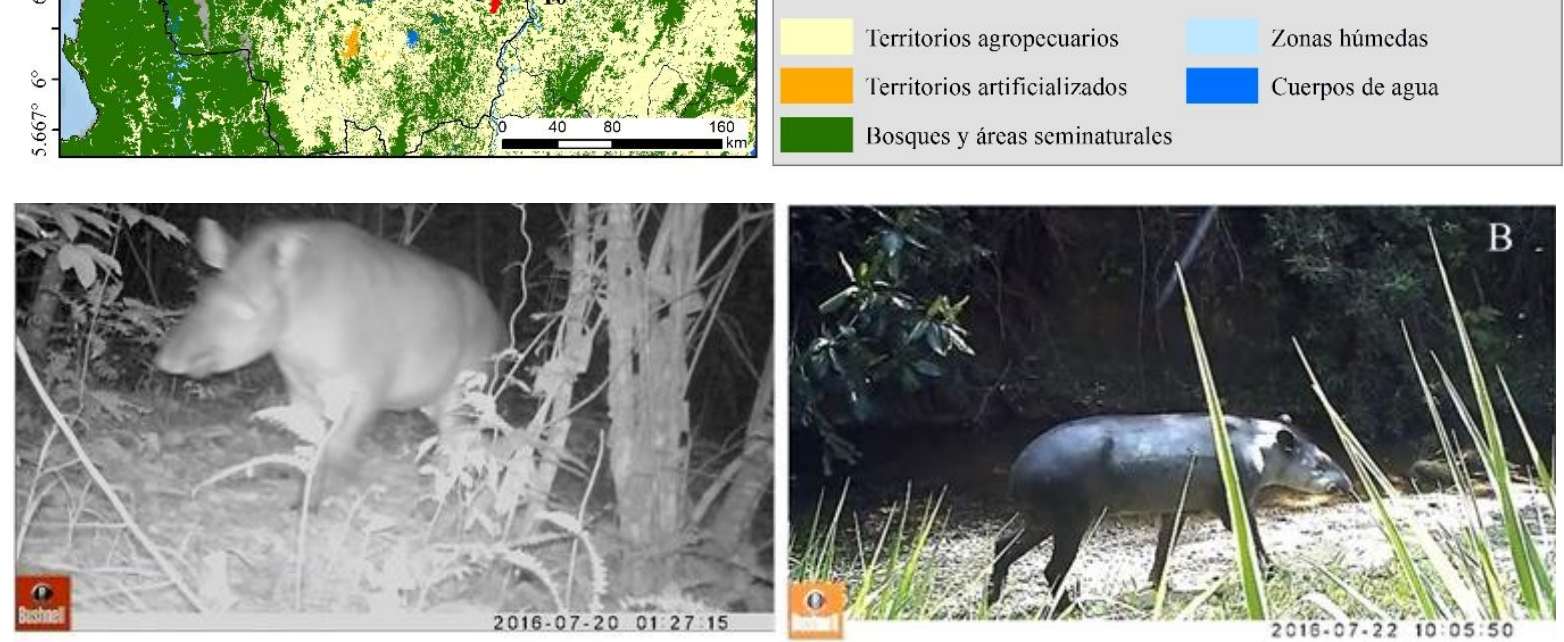

FIGURA 1. A. Ubicación geográfica de los registros de la Danta Colombiana (Tapirus terrestris colombianus). Triángulos, localidades reportadas por Hershkovitz (1954) (*localidad dudosa); cuadrados, localidades reportadas por Arias-Alzate et al. (2009); pentágono, localidad reportada por RaceroCasarrubia \& Arias-Alzate (2015); círculos, localidades aquí registradas. PNN: Parque Nacional Natural, RFPN: Reserva Forestal Protectora Nacional, DRMI: Distrito Regional de Manejo Integrado, DMI: Distrito de Manejo Integrado, RNSC: Reserva Natural de la Sociedad Civil, RN: Reserva natural, SNSM: Sierra Nevada de Santa Marta. B. Individuos de Tapirus terrestris colombianus (izquierda hembra, derecha Macho) registrados en el Magdalena medio antioqueño, Colombia. (Áreas protegidas UNEPWCMC-IUCN 2020; Coberturas de la tierra CLC 2012).

Históricamente, Hershkovitz (1954) reportó la existencia de la Danta Colombiana para el norte de Colombia a partir de 6 localidades, siendo el municipio del Salado, entre los departamentos de Magdalena y La Guajira, la localidad tipo para la subespecie (espécimen descrito en 1942, Holotipo: USNM 281389) (Figura 1A). Es de señalar que, para muchas áreas de esta región, la especie se consideraba extinta localmente, por ejemplo, para la zona del 
alto valle del Río Sinú, departamento de Córdoba (única localidad con presencia histórica de T. terrestris y T. bairdii) (Hershkovitz 1954; Montenegro 2005). Aproximadamente 50 años después de estos reportes documentados en 1954 (los reportes originales datan de mucho más tiempo (e.g., 1829, ver Hershkovitz (1954)), estudios posteriores identificaron y confirmaron la presencia de la especie en las tierras bajas del norte de la Cordillera Central en Antioquia, en límites con la Serranía de San Lucas (Arias-Alzate \& Palacio-V 2004; AriasAlzate et al. 2009). Sin embargo, para la zona del Magdalena medio antioqueño, las poblaciones se consideraban extintas localmente (último registro anecdótico 1997, entrevista), principalmente por la cacería y la pérdida de hábitat (Arias-Alzate \& Palacio-V 2004; Constantino et al. 2006; Arias-Alzate et al. 2009; Varela et al. 2019). Esto ha reducido considerablemente su distribución en la zona central de Colombia. No obstante, estudios recientes confirman su reaparición en el PNN Paramillo, luego de años sin registro en el parque (Racero-Casarrubia \& Arias-Alzate 2015).

Tabla 1. Registros de la Danta Colombiana (Tapirus terrestris colombianus) en el Magdalena Medio antioqueño, Colombia (Datum: WGS84).

\begin{tabular}{cccccc}
\hline Cámara & Sexo & Hora registro & Lat. & Long. & Fecha \\
\hline 1 & Hembra & $02: 46: 47$ & 6,76883 & $-74,380639$ & $20 /$ junio/2016 \\
\hline 2 & Macho & $02: 40: 38$ & 6,792889 & $-74,371528$ & $20 /$ julio/2016 \\
\hline \multirow{3}{*}{3} & $00: 25: 31$ & 6,792889 & $-74,371861$ & $8 / \mathrm{julio} / 2016$ \\
& \multirow{3}{*}{ Hembra } & $04: 04: 03$ & 6,792889 & $-74,371861$ & $13 / \mathrm{julio} / 2016$ \\
& & $01: 27: 15$ & 6,792889 & $-74,371861$ & $20 / \mathrm{julio} / 2016$ \\
\cline { 2 - 6 } & \multirow{2}{*}{ Macho } & $21: 51: 14$ & 6,792889 & $-74,371861$ & $16 / \mathrm{julio} / 2016$ \\
& & $22: 52: 29$ & 6,792889 & $-74,371861$ & $28 / \mathrm{junio} / 2016$ \\
\cline { 2 - 6 } & Indeterminado & $01: 32: 05$ & 6,792889 & $-74,371861$ & $20 / \mathrm{julio} / 2016$ \\
\hline 4 & Macho & $10: 05: 50$ & 6,79475 & $-74,3705$ & $22 / \mathrm{julio} / 2016$ \\
\hline
\end{tabular}

A pesar de que la danta actualmente se considera rara en muchas áreas al norte de su distribución en Colombia, es un ungulado de gran tamaño que por lo general no pasa desapercibida por los pobladores locales. Es una de las especies que presenta grandes presiones por cacería de subsistencia a lo largo de su distribución (Constantino et al. 2006). Esto señala que luego de 19 años sin reportes (desde 1997 a 2016) y de ser considerada extinta para la zona del Magdalena Medio (Arias-Alzate et al. 2009), esta reaparición aquí documentada podría representar dos posibilidades no excluyentes.

Por un lado, a pesar de múltiples esfuerzos de muestreo y monitoreo directo en el nororiente de Antioquia durante los años 2003 y 2005 (Arias-Alzate \& Palacio-V 2004; AriasAlzate 2005), de expediciones realizadas por el primer autor durante el 2008 en los municipios de Maceo y Puerto Berrío, de monitoreos en felinos y sus presas potenciales con cámaras de rastreo en diferentes localidades entre 2010 a 2012 (e.g., Yondó, Puerto Berrío, Maceo, Puerto Nare) (Arias-Alzate et al. 2010, 2012a; Sánchez-Londoño et al. 2011) y de trabajos realizados por otros investigadores en el Bosque Berlín, Puerto Berrío desde 2011 a 2014 (Giraldo \& Álvarez-Hincapié com. pers.) (localidad del registro anecdótico de tapir), no registraron la presencia directa o indirecta de la especie, estos registros podrían representar un posible evento de recolonización reciente en la zona. Esto facilitado probablemente por la recuperación y transformación de las coberturas (i.e., pastos a rastrojos y luego a bosques secundarios) como consecuencia del abandono de tierras por el conflicto 
armado en el área (Marín-Valencia et al. 2018). El registro confirmado más reciente y cercano se encuentra en el municipio de Remedios, Antioquia (observación del año 2003) (Arias-Alzate et al. 2009) a aproximadamente 21,5 km de distancia (Figura 1A).

Por otro lado, aunque los tapires dependen considerablemente de las coberturas boscosas y no toleran fuertes procesos de fragmentación y cacería (Varela et al. 2019) (como los ocurridos en el Magdalena Medio antioqueño (Etter \& Rodríguez 2008; Marín-Valencia et al. 2018), es posible que la especie no se extinguiera localmente y ha pasado desapercibida durante años. Esto debido a las bajas densidades poblacionales causadas por las presiones antrópicas locales. Por ejemplo, se han propuesto diferentes estimaciones de densidad que van desde 0,2 ind $/ \mathrm{km}^{2}$ en áreas muy fragmentadas, hasta 3,7 ind $/ \mathrm{km}^{2}$ en áreas con un buen estado de conservación como la Amazonía (Mendes-Pontes 2004; Ferreguetti et al. 2017; Varela et al. 2019). Futuros estudios permitirán dilucidar la viabilidad y el estado actual de estas poblaciones, las cuales posiblemente se encuentren en mejor estado hacia zonas con mayor cobertura como la Serranía de San Lucas (Figura 1A).

Actualmente la subespecie es la menos representada en el Sistema Nacional de Áreas Protegidas de Colombia, siendo el PNN Paramillo la única área protegida cercana con presencia confirmada (ubicada a $\sim 172 \mathrm{~km}$ de distancia) (Racero-Casarrubia \& Arias-Alzate 2015). Adicionalmente, en la zona de amortiguamiento del PNN SNSM, en la reserva local Buena Vista, en el municipio de Dibulla se hacen algunos esfuerzos por su conservación (Florez et al. 2008). Sin embargo, esta baja representatividad en pocas áreas de protección en el centro-norte del país, señalan la problemática que afronta la especie en términos de conservación en el futuro cercano. Los continuos procesos de fragmentación y transformación de los remanentes de bosque nativo en el Nororiente de Antioquia, continúan poniendo en peligro de extinción no solo a la Danta Colombiana, sino también a otras especies sombrilla, como el Jaguar (Panthera onca), el Puma (Puma concolor) y el Oso de Anteojos (Tremarctos ornatus) (Arias-Alzate et al. 2013; Vela-Vargas et al. 2014). Por ello, es de gran importancia la creación de un área protegida de carácter nacional que salvaguarde estos hábitats nativos en la zona.

En este sentido, la Serranía de San Lucas con 9.397 km² (González-Maya et al. 2011) y catalogada como zona prioritaria en Colombia (IAvH et al. 2008), representa un área de gran relevancia para la conservación de estas especies, al ser una zona estratégica que conecta varios ecosistemas. Por ejemplo, al suroccidente se conecta con la Reserva forestal Bajo Cauca-Nechí en Antioquia, estas a su vez se conectan al occidente del país con el PNN Paramillo, las Selvas inundables del Urabá, las Selvas del Darién y el PNN Los Katíos en los departamentos de Antioquia y Chocó. Todas son zonas que albergan una gran diversidad de especies y sirven como corredores de acceso y conexión entre Centro y Sur América (Arias-Alzate et al. 2012b; Hernández-Camacho et al. 1992). Es de resaltar que cerca del área donde se encontraron los registros documentados por este estudio y al suroriente de la Serranía de San Lucas se encuentra el DRMI Ciénaga de Barbacoas y la RNSC San Bartolo (Figura 1A), zonas que requieren de una mayor atención.

Esperamos que estos hallazgos ayuden a entender mejor la presencia actual de esta especie en la zona y pueden ser un punto de partida para apoyar estudios futuros a largo plazo en aspectos de ocupación, densidad poblacional e historia natural. Igualmente recalcamos su importancia y su potencial como sustento para el establecimiento de la Serranía de San Lucas como un área protegida de carácter nacional, de gran importancia en 
términos de conservación de ésta y otras especies en riesgo de extinción, de los ecosistemas y sus procesos ecológicos no solo en el Magdalena Medio antioqueño, sino también en la región.

\section{AGRADECIMIENTOS}

Agradecemos a los propietarios de la Agro-ganadería Montenegro por permitir los monitoreos en la zona. Igualmente agradecemos al coordinador del equipo fauna-CORANTIOQUIA, Juan Camilo Restrepo Llano por suministrarnos y permitirnos documentar esta información. Agradecemos a dos revisores anónimos por sus valiosos comentarios.

\section{REFERENCIAS}

Arias-Alzate A, Palacio-V JA. 2004. Búsqueda de poblaciones y algunos aspectos de la utilización del Hábitat de la Danta Colombiana Tapirus terrestris colombianus en el nordeste antioqueño. Informe final. Corporación Autónoma Regional Del Centro De Antioquia CORANTIOQUIA.

Arias-Alzate A, Palacio Vieira JA, Muñoz-Durán J. 2009. Nuevos registros de distribución y oferta de hábitat de la Danta Colombiana (Tapirus terrestris colombianus) en las tierras bajas del norte de la Cordillera Central (Colombia). Mastozoolgía Neotropical 16:19-25.

Arias-Alzate A, Sánchez-Giraldo C, Botero CS, Solari S. 2010. Aproximación al estado de conservación de las especies de felinos en algunos municipios pertenecientes a las cuencas aportantes del sector eléctrico en jurisdicción de Corantioquia. Informe Final Corporación Autónoma Regional Del Centro De Antioquia CORANTIOQUIA.

Arias-Alzate A, Botero-Cañola S, Sánchez-Londoño JD, Solari S. 2012a. Caracterización del estado de los felinos (Carnivora: Felidae) y su interacción con el hombre en el oriente de Antioquia. informe final Corporación Autónoma de las Cuencas de los Ríos Negro y Nare, CORNARE.

Arias-Alzate A, González-Maya JF, Víquez-R LR. 2012b. Conservación de mamíferos del Chocó: distribución, riqueza y representatividad de las áreas protegidas. Revista Biodiversidad Neotropical 2:71-82

Arias-Alzate A, Botero-Cañola S, Sánchez-Londoño JD, Solari S. 2013. Presencia de felinos y evidencias de conflicto con humanos en tres regiones de Antioquia. En: Payán Garrido E, CastañoUribe C, editores. Grandes Felinos de Colombia, Vol. I. Fundación Herencia Ambiental Caribe, Conservación Internacional, y Cat Specialist Group UICN/SSC. Bogotá, D. C., Colombia.

Coberturas de la tierra, Corine land Cover (CLC) Período 2005-2009, escala 1:100.000. 2012. Sistema de información ambiental de Colombia-IDEAM. http:/ / www.siac.gov.co/catalogo-de-mapas

Constantino E, Lizcano D, Montenegro O, Solano C. 2006. Danta común. Tapirus terrestris. En: Rodríguez-Mahecha JV, Alberico M, Trujillo F, Jorgenson J, editores. Libro rojo de Los Mamíferos de Colombia, Serie Libros Rojos de Especies Amenazadas de Colombia. Conservación Internacional Colombia, Ministerio del Medio Ambiente, Vivienda y Desarrollo Territorial. Bogotá, Colombia. Bogotá, Colombia. p. 108-115.

Etter A, Rodríguez N. 2008. Transformación de la cobertura forestal en el Valle del Magdalena Medio en el periodo de 1987-2001. Ambiente y Desarrollo 12:105-132.

Ferreguetti ÁC, Tomás WM, Bergallo HG. 2017. Density, occupancy, and detectability of lowland tapirs, Tapirus terrestris, in Vale Natural Reserve, southeastern Brazil. Journal of Mammalogy 98: 114-123. https://doi.org/10.1093/jmammal/gyw118 
Florez FK, Rueda CF, Peñalosa W, Rodríguez J, Torres G, Armenta MM. 2008. Distribución histórica y actual de la población de danta de tierras bajas Tapirus terrestris colombianus (Hershkovitz 1954) más al norte de Sur América. Tapir Conservation 17:22-25.

González-Maya JF, Balaguera S, Zarrate-Charry DA. 2011. Construcción Mosaico de Conservación de la Serranía de San Lucas. Informe Técnico Final. ProCAT Colombia-UAESPNN -CAF. Bogotá, Colombia.

Hernández-Camacho JI, Hurtado Guerra A, Ortiz Quijano R, Walschburger T. 1992. Unidades biogeográficas de Colombia. Acta Zoológica Mexicana Vol. Espec:105-151.

Hershkovitz P. 1954. Mammals of the northern Colombia, preliminary report No.7: Tapirs (Genus Tapirus), with a systematic review of American species. Proceedings of the United States National Museum 103:465-496. https:// doi.org/10.5479/si.00963801.103-3329.465

Holdridge LR 1947. Determination of world plant formations from simple climatic data. Science 105:367-368. https:// doi.org10.1126/science.105.2727.367

Instituto de Investigación de Recursos Biológicos Alexander von Humboldt, SIRAP, The Nature Conservancy, editors. 2008. Acta de compromiso entre la mesa de trabajo del sistema regional de área protegidas del Caribe colombiano, el Instituto de Recursos Biológicos Alexander Von Humboldt y The Nature Conservancy, Planificación ecorregional para la definición de áreas protegidas para la conservación de la biodiversidad en el área de jurisdicción de la mesa SIRAP Caribe, Informe técnico.

Marín-Valencia AL, Álvarez-Hincapié CF, Giraldo CE, Uribe-Soto S. 2018. Análisis multitemporal del paisaje en el Magdalena Medio en el periodo 1985-2011: una ventana de interpretación de cambios históricos e implicaciones en la conectividad estructural de los bosques. Cuadernos de Geografia: Revista Colombiana de Geografia 27:10-26.

https://dx.doi.org/10.15446/rcdg.v27n1.55783

Mendes-Pontes AR. 2004. Ecology of a community of mammals in a seasonally dry forest in Roraima, Brazilian Amazon. Mammalian Biology 69:319-336. https:// doi.org/10.1078/1616-5047-00151

Ministerio de Ambiente y Desarrollo Sostenible. Resolución 0119 de 2017. Listado de las especies silvestres amenazadas de la diversidad biológica colombiana continental y marino costera. Bogotá: MADV. Disponible en: http:/ / www. minambiente.gov.co/images/normativa/app/resoluciones/75-res\%201912\%20de\%202017.pdf

Montenegro O. 2005. Programa nacional para la conservación del género Tapirus en Colombia. Ministerio de Ambiente, Vivienda y Desarrollo Territorial. Dirección de Ecosistemas. Bogotá, Colombia.

Padilla M, Dowler R. 1994. Tapirus terrestris. Mammalian Species 481:1-8. https:// doi.org/10.2307/3504109

Racero-Casarrubia J, Arias-Alzate A. 2015. Presencia y primeras fotografias del Tapir de Tierras Bajas (Tapirus terrestris) en el Parque Nacional Natural Paramillo, Córdoba, Colombia. Tapir Conservation 24(33):4-6

Sánchez-Londoño JD, Arias-Alzate A, Botero-Cañola S, Solari S. 2011. Seguimiento del Estado de las Poblaciones de Grandes Felinos, así como la Interacción de estos con los Pobladores y sus Actividades Productivas en el Magdalena Medio Antioqueño. Informe final Corporación Autónoma Regional del Centro de Antioquia, CORANTIOQUIA 
Varela D, Flesher K, Cartes JL, De Bustos S, Chalukian S, Ayala G, Richard-Hansen C. 2019. Tapirus terrestris. The IUCN Red List of Threatened Species 2019: e.T21474A45174127. https:// dx.doi.org/10.2305/IUCN.UK.2019-1.RLTS.T21474A45174127.en. Downloaded on 23 May 2020.

Vela-Vargas IM, González-Maya JF, Pineda-Guerrero A, Zárrate-Charry D. (2014). Primer registro confirmado de Oso Andino (Tremarctos ornatus: Ursidae) en la Serranía de San Lucas, Bolivar, Colombia. Mammalogy Notes 1:11-12.

UNEP-WCMC, IUCN. 2020. Protected Planet: The world database on Protected Areas (WDPA)/. Annual release 2020. Cambridge, UK: UNEP-WCMC and IUCN. Available at: www.protectedplanet.net.

Editor: Diego J. Lizcano Recibido: 2020-04-29

Revisado: 2020-05-25

Aceptado: 2020-06-07

Publicado: 2020-06-26 